\title{
The Human Mesenchymal Stem Cells and the Chick Embryo Chorioallantoic Membrane: The Key and the Lock in Revealing Vasculogenesis
}

\author{
ŞERBAN COMŞA ${ }^{1}$, RALUCA AMALIA CEAUȘU ${ }^{1}$, ROXANA POPESCU ${ }^{2}$, \\ ANCA MARIA CÎMPEAN ${ }^{1}$ and MARIUS RAICA ${ }^{1}$ \\ ${ }^{1}$ Department of Histology, Angiogenesis Research Center, ${ }^{2}$ Department of Cell and Molecular Biology, \\ "Victor Babeş" University of Medicine and Pharmacy, Timisoara, Romania
}

\begin{abstract}
Background/Aim: To analyze the interaction between the human mesenchymal stem cells (hMSC) and the chick embryo chorioallantoic membrane (CAM), in order to assess the still obscure process of vasculogenesis. Materials and Methods: We implanted hMSC onto CAM and we analyzed the morphology and the immunohistochemical profile of CAM. Results: hMSC adhered to CAM, few of them entered the chorionic epithelium and the mesoderm and developed a CD44-/Ki67- status. hMSC stimulated the CAM mesenchymal cells (cMSC) to acquire endothelial and pericyte-like features and to generate cord/capillary-like structures (CLS) in the chorionic epithelium and the mesoderm, but they also entered these structures $\left(C D 34^{+} / S M A\right.$ (smooth muscle actin) + hMSC). Simultaneously, hMSC induced a process of sprouting angiogenesis in the mesoderm, CD105 ${ }^{+}$hMSC being identified in the proximity of the angiogenic areas. Conclusion: hMSC and CAM establish a genuine hotspot of vasculogenesis, which may evolve to a valuable experimental model for this research field.
\end{abstract}

As the vascular system participates in the pathogenesis of many diseases, such as cancer, stroke, and myocardial infarction, it is essential to elucidate the molecular mechanisms of blood vessel formation to better understand and manipulate the pathological mechanisms involved (1).

This article is freely accessible online.

Correspondence to: Anca Maria Cîmpean, "Victor Babeş" University of Medicine and Pharmacy, Department of Histology, Piaţa Eftimie Murgu nr. 2, 300041, Timişoara, Timiş, Romania. Tel: +40 720060955, Fax: +40 256490626, e-mail: ancacimpean1972@ yahoo.com

Key Words: Angiogenesis, chorioallantoic membrane, mesenchymal stem cells, pericyte-like, vasculogenesis.
Vasculogenesis entails the direct formation of blood vessels by differentiation of angioblastic precursor cells in situ, while angiogenesis entails new vessel formation from preexisting vessels, capillaries and postcapillary venules (2). The first blood vessels which appear during embryogenesis are generated by vasculogenesis. Essential steps in this process are the establishment of the endothelial cell lineage (angioblasts), the assembly of angioblasts into cord-like vascular structures, the formation of vascular lumens, and the organization of vascular networks (3).

Vasculogenesis was previously considered to occur primarily during physiological development of vasculature, but recent evidence indicates that endothelial progenitor cellmediated vasculogenesis also contributes to pathological neovascularization (4). Tumor vasculature, for instance, has been thought to emerge by expansion of the host vasculature through postnatal sprouting of endothelial cells (angiogenesis). Therefore, it was proposed that inhibiting angiogenesis would stop tumor growth, but when this conceptually simple objective turned out to be impossible to reach, a notion of neo-vasculogenesis was born, suggesting that tumor vessels develop from circulating bone marrowderived endothelial precursors rather than from mature endothelial cells (5).

The chick embryo chorioallantoic membrane is an extraembryonic membrane that is commonly used in vivo to study both angiogenesis and anti-angiogenesis (2), but nonembryonic vasculogenesis was seldom studied onto this model. Its blood vessels appear at day 4 of incubation as a network of primitive, irregularly spaced tubules with large luminal diameters, scattered in the mesodermic layer, while from days 5 to 10 , CAM vessels progressively differentiate into capillaries, arterioles and venules (6). Starting from day 10, CAM may be used as a model for vasculogenesis induction, the interference of the embryonic vasculogenesis being excluded. 
The angiogenic potential of hMSC is an area of current and increasing interest. hMSC have proven their involvement in promoting angiogenesis following tissue injury in several disease models (7) and have recently been associated with vasculogenesis, as they were able to differentiate into endothelial cells and contribute to in vivo neovascularization, under optimized conditions (8).

Taking into account this context, we aimed to analyze the interaction between hMSC and CAM, in order to assess the still obscure process of vasculogenesis. To our knowledge, this is the first report describing the direct interaction between hMSC and CAM.

\section{Materials and Methods}

hMSC cell culture. hMSC were purchased from Cellular Engineering Technologies Inc. (Coralville, USA) and had the previously evaluated molecular profile (flowcitometry): $\mathrm{CD}_{105^{+}}$, $\mathrm{CD}^{2} 0^{+}, \mathrm{CD}_{4} 4^{+}, \mathrm{CD} 29^{+}, \mathrm{CD}_{4}{ }^{-}, \mathrm{CD} 14^{-}$. hMSC were culture expanded in low-glucose DMEM and 20\% Fetal Bovine Serum, supplemented with $25 \mathrm{ng} / \mathrm{ml}$ basic fibroblast growth factor and trypsinized when they reached a confluence of $70-80 \%$. hMSC were reseeded at $2-3 \times 10^{3} / \mathrm{cm}^{2}$ in T75 flasks, filled with $12 \mathrm{ml}$ of culture medium. Cells from passage 4 were used in experiments.

CAM experimental model preparation. A total of 20 fertilized chicken eggs were incubated at $37^{\circ} \mathrm{C}$. On day 3 of incubation, a window in the eggs' shell was cut and it was consecutively covered with Parafilm. The eggs were then reincubated for another 7 days.

Implantation of hMSC onto CAM. A ring of silicone was positioned onto the CAM surface of each egg, under sterile conditions, on day 10 of incubation (day 1 of the experiment). The eggs were divided into two subsets: 5 were part of the control group and 15 part of the experimental group. $5 \mu \mathrm{l}$ of DMEM were added inside the rings of the control eggs while the eggs from the experimental group received a suspension of hMSC $\left(2 \times 10^{4} / 5 \mu \mathrm{l}\right.$ DMEM). The experiment ended 7 days later (day 17 of incubation) through the in ovo instillation of 5 $\mathrm{ml}$ formalin $4 \%$, onto the surface of the CAM. During the incubation, the viability of the eggs was assessed daily. The eggs had to be viable until the end of the experiment in order to be subject of the following CAM examination.

Stereomicroscopic evaluation. After the implantation of hMSC onto CAM, the eggs were evaluated using a Zeiss stereomicroscope (Axio Zoom.V16) on days 2, 4 and 7 of the experiment (days 11, 13 and 16 of incubation).

Morphological evaluation. For the morphological evaluation, each egg's CAM was detached and submerged for fixation in $4 \%$ formalin solution, for $48 \mathrm{~h}$. Consecutively, the paraffin embedding was practiced, then $5 \mathrm{~mm}$-thick sections were cut by the microtome and hematoxylin and eosin staining was finally performed.

Immunohistochemical expression. The incubation step of the immunohistochemical technique was performed for $15 \mathrm{~min}$, by using the following Bond ready-to-use primary antibodies for the specific human markers: SMA (clone $\alpha$ sm-1), Ki67 (clone K2), CD44 (clone
VFF-8), CD105 (clone 4G11), CD34 (clone QBEnd/10) from Leica Biosystems Newcastle Ltd, Newcastle uponTyne, Britain) and chick/human CD34 from Abbiotec, San Diego, USA. Bond Polymer Refine Detection System (Leica) was used for visualization. The chromogen dihidrochlorid-3, 3-diamine-benzidine was applied for 10 min. Lille's hemotoxylin - $5 \mathrm{~min}$, was used as counterstain. The entire procedure was performed with the Leica Bond-Max autostainer (Leica Biosystems, uponTyne Newcastle, UK).

The immunoexpression was evaluated at nuclear level for Ki67, cytoplasmic level for SMA and CD34 and membranous level for CD105 and CD44. The intensity of the immunohistochemical reaction was classified as it follows: negative $(-)$, low $(+)$, moderate $(++)$, high $(+++)$.

The morphological and immunohistochemical slides were evaluated using a Zeiss Axio Imager 2 light microscope and the images were recorded using the Zeiss Efficient Navigation Software.

\section{Results}

Stereomicroscopic evaluation. Starting from day 2 of the experiment, the evaluation of the eggs' CAM onto which MSC were added, revealed a clear tendency for MSC migration. In addition, they demonstrated a tendency to organize into large cord-like structures onto CAM, inside and outside the silicon ring they had been added to, with a clear affinity for ring adherence. By day 7 of the experiment, MSC developed clusters onto CAM and stimulated an intense sprouting angiogenesis, starting from the large mesodermic vessels. No such images could be observed in the control eggs.

At the end of the experiment, 3 out of the 5 control eggs and 10 out of the 15 experimental eggs were still viable. These eggs were the subject of the following evaluation.

Morphological evaluation. In the control group, there was a vascular sinus development in the mesoderm, in the proximity of the chorionic epithelium, but it was not filled with blood cells until day 17 of incubation. Capillaries, filled with blood cells, were to be identified in intimate contact with the sinus cavities, showing a tendency to merge with them. Strands of tissue were still present in some areas separating the cavities of the sinus, while in some zones prolongations of the delineating cells proved a tendency to partition the sinus by forming cytoplasmic pillars.

In the experimental group, hMSC adhered to the chorionic epithelium and stimulated a clear process of sprouting angiogenesis in the mesoderm, starting from the existing vessels, related to the areas of hMSC agglutination onto the CAM surface. In addition, they determined the appearance of an intense vasculogenic reaction in the chorionic epithelium and the mesoderm, by stimulating cMSC to develop long and thin prolongations through which they consecutively connected to neighboring cMSC and formed cord-like and, in some areas, capillary-like structures (Figure 1a and b). The connection between CLS and the large vessels of the mesoderm was not constantly evident, confirming that CLS 

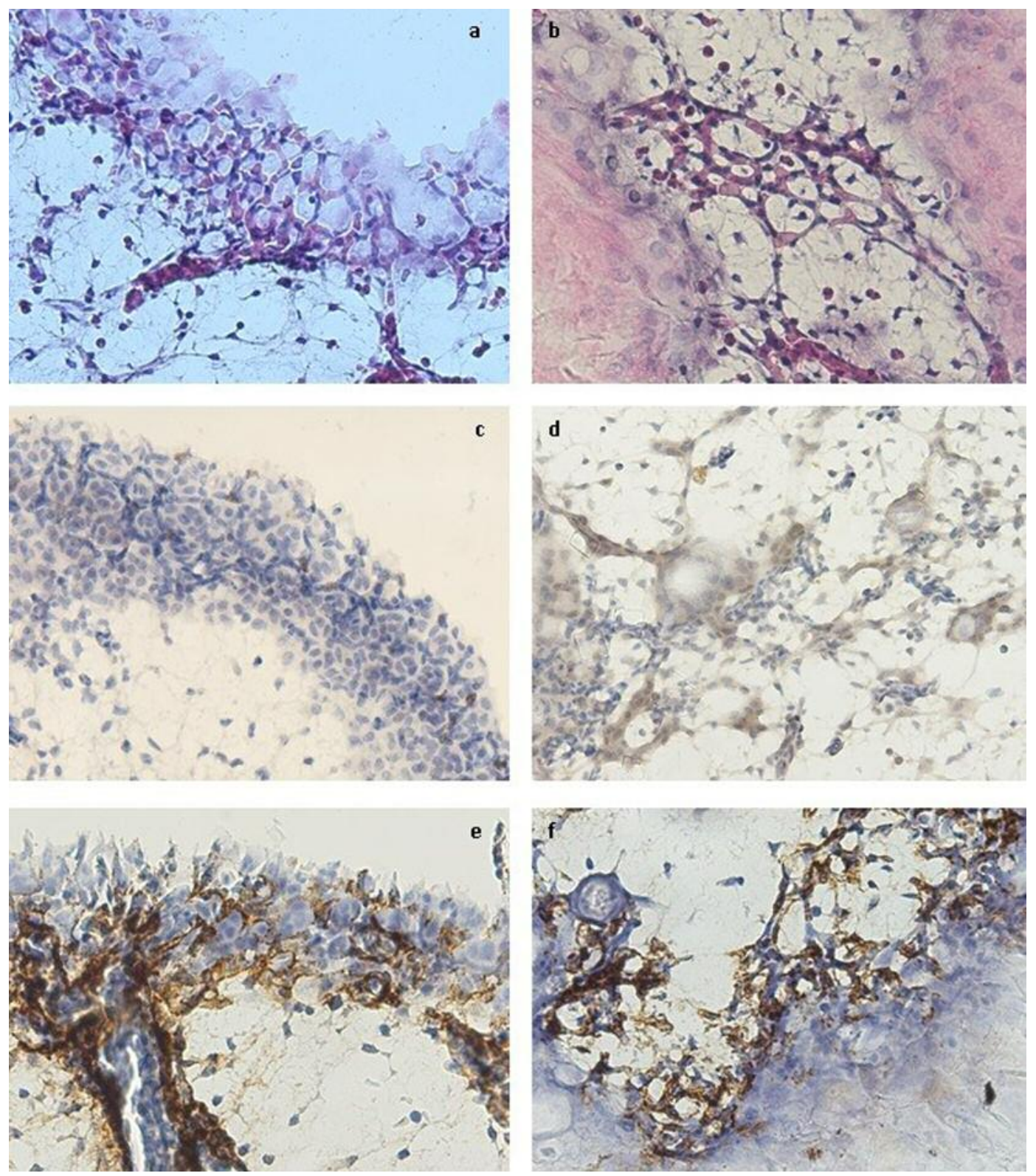

Figure 1. The morphology and immunohistochemical profile of the cord/capillary-like structures from the chorionic epithelium (a, $c$, e) and the mesoderm $(b, d, f) . a, b-$ morphology (hematoxylin-eosin staining); $c-h C D 34$ expression; $d-c C D 34$ expression; e, $f-S M A$ expression. Magnification $\times 400$.

formed through vasculogenesis. There was also a concomitant differentiation of cMSC towards blood cell lineage, inside the capillary-like structures, suggesting that, onto CAM, vasculogenesis and hematopoiesis are co-localized and concomitant processes. The vessel lumen formation mechanism seemed to be a combination between cord and cell hollowing. On the other hand, there was no evidence of a blood sinus formation beneath the chorionic epithelium.
Immunohistochemical evaluation. The adherent hMSC to the CAM surface had an isolated low CD105 expression, but mostly negative. A similar condition was also noticed in the chorionic epithelium. Interestingly, there were few CD105 moderate positive hMSC inside the mesoderm, in close relation with the angiogenic areas, suggesting that their penetration towards the mesoderm 'saves' their endoglin expression and, also, their angiogenic potential. The CD44 
expression was negative both in the hMSC from the CAM surface and in the hMSC situated inside the CAM structures. Ki67 was also negative, suggesting that hMSC have a nonproliferative status either if they reside onto CAM, or if they enter the chorionic epithelium or the mesoderm.

The hCD34 marker had an isolated moderate positive expression in the areas of vasculogenesis, more often in the chorionic epithelium than in the mesoderm, suggesting the involvement of the CD34 ${ }^{+} \mathrm{hMSC}$ in this process (Figure 1c). The cCD34 marker was diffusely expressed in all the vasculogenic areas, especially associated with the cMSC from the mesoderm, but the intensity of the expression was constantly low (Figure 1d). hMSC from the CAM surface expressed neither human nor chicken/human CD34.

Although isolated, both hCD34 and CD105 expressions suggest the ability of hMSC to cross the chorionic epithelium and to enter the mesoderm, but also their involvement in the local angiogenic and vasculogenic processes.

The SMA expression was extensive in the cMSC from the mesoderm, but the intensity was generally low. In the periphery of the large vessels from the mesoderm, there was an intense reaction, suggesting a high expression of this marker. A similar pattern was identified in the angiogenic and vasculogenic areas from the mesoderm and the chorionic epithelium. The $\mathrm{SMA}^{+}$cells were in close contact with the cord- and capillary-like structures, covering even the segments with no visible lumen, and, in some areas, they were even involved in the cord-like structure formation. The morphology and the localization of the $\mathrm{SMA}^{+}$cells are suggestive for including these cells in the hMSC and the cMSC types (Figure $1 \mathrm{e}, \mathrm{f}$ ).

In the control group, SMA was intensely expressed, but limited to the periphery of the large vessels of the mesoderm, while CD105, CD44, hCD34 and cCD34 were all negative.

\section{Discussion}

Vasculogenesis is a very complex process involving several molecular and cellular mechanisms, while MSC represent a controversial cell line, able to modulate this process through their versatile behavior facilitated by the in vivo context.

The implantation of MSC onto CAM proves to be a valuable experimental model, useful in answering to specific questions regarding the involvement of hMSC in vasculogenesis. The presence of hMSC onto CAM induced morphological and immunohistochemical changes in both hMSC and CAM, demonstrating the mutual influence they exercise onto each other.

Onto CAM, hMSC changed their molecular profile, as they totally lost the expression of CD44 and, partially, the expression of CD105. Interestingly, some of the hMSC which managed to enter the mesoderm kept the CD105 expression. CD44 is a well-described adhesion molecule existing in different isoforms that interact with multiple ligands, such as hyaluronan, selectins, collagen and fibronectin, but the functional consequence for acquisition of CD44 expression on the expanded MSC during therapeutic use remains unclear (9). Qian et al. provide functional and molecular evidence suggesting that MSC physiologically do not express CD44 in both humans and mice and confirm that in vitro culture could result in acquisition of CD44 expression on their surface and changes in the expression of cytokines, growth factors, matrix proteins and other signaling molecules (9). In this context, the absence of the CD44 expression in MSC, after implantation onto CAM, represents a proof that MSC regain their precultivation CD44 status when they re-enter the in vivo medium.

Regarding CD105, Anderson et al. consider that its expression on murine MSC is heterogeneous, which is in agreement with previous studies on murine MSC and human MSC (10). It has been proven that CD105 is fundamental for the development and function of the cardiovascular system and has been implicated in angiogenesis (11). It has generally been used to select for multipotent human MSC, while its disappearance has been associated with MSC differentiation (12). Our results for CD105 expression demonstrate that the CAM surface may stimulate MSC to enter the differentiation process, while the cells that reach the chorionic epithelium and the mesoderm maintain their multipotency and their implication in angiogenesis or vasculogenesis. Levi et al. demonstrated that adipose tissue-derived MSC with low expression of CD105 presented a significantly enhanced in vitro osteogenic differentiation potential (13), so we might hypothesize that MSC from the CAM surface could differentiate towards the osteoblastic line. This hypothesis is supported by the involvement of the chorionic epithelium in the embryonic osteogenesis, as it contains the calciumtransporting region of the CAM, which facilitates the transport of calcium from the eggshell into the embryo (6).

Surprisingly, in our study we noticed the appearance of a both CD44 and CD105 negative phenotype in MSC after implantation onto CAM, but this phenotype has also been suggested by Qian et al., as they demonstrated that freshly sorted CD44- MSC displayed low expression for CD105, showing a discrepancy from what has been reported for cultured MSC. They concluded that CD105 is up-regulated on the cultured MSC, as compared with the freshly sorted CD44- MSC (9).

CD34 is predominantly regarded as a marker of hematopoietic stem cells and hematopoietic progenitor cells. However, CD34 is now also established as a marker of several other non-hematopoietic cell types, including MSC or vascular endothelial progenitors (14). Lin et al. suggest that available evidence points to CD34 being expressed in tissue-resident MSC and its negative finding being a consequence of cell culturing. In addition, CD34 expression 
correlates with enhanced vasculogenic and angiogenic potential, but also with replicative capacity and stemness, while loss of CD34 expression might be related to lineage commitment (15). In our study, we evaluated the CD34 expression using two antibodies, one for chicken/human CD34 (cCD34) and another one for human CD34 (hCD34). CD34 presented different expression intensities and localization, depending on the antibody we used. hCD34 had a moderate, but isolated expression, more often at the level of the chorionic epithelium, while the cCD34 expression was low, but diffusely positive especially in the mesodermic mesenchymal tissue. These results suggest that the antibody for hCD34 is more sensitive for the identification of the $\mathrm{CD} 34^{+}$hMSC than the cCD34 variant. On the other hand, the CD34 expression could translate the acquisition of a more vasculogenic status in hMSC than in the cMSC, in the context of the MSC/CAM interaction. Both CD34 expressions were, anyway, associated with the vasculogenic areas, confirming the above conclusions of Lin et al. In addition, the discrepancy in CD34 expression in the hMSC population (CD34- $4^{-}$MSC on the CAM surface and CD34 ${ }^{+}$ hMSC inside the CAM structures), sustains the previous findings regarding the influence of the environment on the CD34 expression: onto CAM surface hMSC still keep the in vitro $\mathrm{CD} 34^{-}$status, while those entering $\mathrm{CAM}$ switch to the tissue -resident cells' CD $34^{+}$status.

Although their vasculogenic and angiogenic potential of the hMSC is evident onto CAM, with an endothelial-like newly established profile, hMSC prove a non-proliferative status, as determined by the Ki67 lack of expression. To our knowledge, there is no previous evaluation of the hMSC proliferative status onto CAM, using the Ki67 status.

It was previously demonstrated that SMA is present in the CAM capillary plexus, and its expression is intimately related to its endothelial layer at least after day 12 of incubation (16). Although the anti-SMA antibody was human specific, it was clearly positive in a population of cMSC and the chick pericytes. It was no surprise to notice that the SMA expression intensity was high in the periphery of the large vessels of the mesoderm, because it was already stated that from days 5 to 10 , CAM vessels progressively differentiate into capillaries, arterioles, and venules (6), so that in the 17th day of incubation all the large mesodermic vessels are mature, so that they contain a layer of $\mathrm{SMA}^{+}$pericytes. What impresses in the SMA is its high expression in the angiogenic and vasculogenic areas, as it proves the close contact of the pericyte-like cells with the new capillaries, but also with CLS, even if they do not present a lumen yet. It is clear that SMA is needed for the stability of the pseudopode like projections present in the pericytes (16), offering a consecutive stability to the vessel wall, but the question is why is this SMA expressed so early in the cMSC? It was hypothesized that pericytes are recruited towards the capillary plexus early in CAM development (before day 10 of incubation) (16) and VEGF seems to stimulate this process (17). Our results regarding the hMSC-stimulated vasculogenic process prove something else. The capillarylike and the cord-like structures are formed by $\mathrm{CD} 34^{+}$cells (in some areas even by $\mathrm{SMA}^{+}$cells) and they are strengthened by $\mathrm{SMA}^{+}$cells layed on the periphery of these structures. All these cells show a mesenchymal morphology, so that we suggest that the mesenchymal stem cell represents the common progenitor for both $\mathrm{CD}_{3} 4^{+}$and $\mathrm{SMA}^{+}$cells involved in the new vessel formation. This idea is sustained by the results of Hagedorn et al. who promote the idea of a synchronized differentiation of the different cellular components of blood vessels, as they observed that after the VEGF stimulation of embryonic bodies, groups of cells expressing both endothelial and pericyte antigens got engaged in a cord formation process (17).

CAM is a frequently used tissue for studying vascular growth and remodeling, notably non-sprouting angiogenesis by formation of transluminal pillars (16), while VEGF seems to be involved in the augmentation of the intussusceptive microvascular growth (17). Ribatti et al. suggests that sprouting angiogenesis may be identified in CAM in the early phase (days 5-7 of development), the later phases being characterized by the appearance of the intussusceptive angiogenesis and the absence of the sprouts (2). Although angiogenic, hMSC induce a sprouting angiogenesis, so that, probably, they act through a different pathway than VEGF. Also, suggestive for this issue is the fact that VEGF stimulates only angiogenic, but not vasculogenic processes in the 17-day old CAM. Biomicroscopy image analysis of control CAM at day 17 showed a typical hierarchical organization of the vascular tree, whereas VEGF perturbed this defined architecture by inducing new capillaries, which emerge out of arterioles, venules, and existing capillaries (17). However, in a previous in vitro model, we demonstrated that hMSC developed long and thin branches interconnecting each other and had a clear tendency to organize in clusters and to form capillary-like structures following exposure to VEGF (18), so that we cannot totally exclude VEGF out of the vasculogenic pathway. It remains to determine the panel of mechanisms activated by hMSC in the CAM nonembryonic vasculogenesis and to evaluate the level of the VEGF involvement in this process.

Strassburg et al. demonstrated that when administered onto CAM, adipose-derived stem cells (ASCs) successfully guided host vasculature into the fibrin construct they applied onto CAM (angiogenesis) and guided formation of capillarylike structures by co-implanted human endothelial cells (vasculogenesis), leading to a perfused avian and human capillary network within the fibrin construct (19). We have confirmed the possibility of a hybrid, human-avian capillary network formation, but we have gone further, as we 
demonstrated that such a capillary network can be developed through the hMSC-cMSC cooperation, with no mature or endothelial cell progenitor involvement.

\section{Conclusion}

The MSC-CAM interaction may be defined as a mutual process. Onto CAM, hMSC induce vasculogenesis, switch to an endothelial-like non-proliferative phenotype and return to the CD44 native negative status. In turn, hMSC stimulate cMSC to acquire a vasculogenic and pericyte-like phenotype: they organize into CLS and start expressing cCD34 and SMA in the vasculogenic areas. hMSC and CAM establish a genuine hotspot of vasculogenesis, which may evolve to a valuable experimental model for this research field.

\section{Acknowledgements}

This work was supported by the PROGRAM II - C3 - TC - 2015 of the "Victor Babeş" University of Medicine and Pharmacy, Timişoara, Romania.

\section{References}

1 Lammert E and Axnick J: Vascular lumen formation. Cold Spring Harb Perspect Med 2: 1-10, 2012.

2 Ribatti D, Nico B, Vacca A, Roncali L, Burri PH and Djonov V: Chorioallantoic membrane capillary bed: A useful target for studying angiogenesis and anti-angiogenesis in vivo. Anat Rec 264: 317-324, 2001.

3 Drake CJ and Fleming PA: Vasculogenesis in the day 6.5 to 9.5 mouse embryo. Blood 95: 1671-1679, 2000.

4 Longeras R, Farjo K, Ihnat M and Ma J: A PEDF-derived peptide inhibits retinal neovascularization and blocks mobilization of bone marrow-derived endothelial progenitor Cells. Exp Diabetes Res 2012: 518426, 2012.

5 Witkiewicz H, Oh P and Schnitzer JE: II. Capsular vaso-mimicry formed by transgenic mammary tumor spheroids implanted ectopically into mouse dorsal skin fold: implications for cellular mechanisms of metastasis. F1000Res 2: 9, 2013.

6 Gabrielli MG and Accili D: The chick chorioallantoic membrane: A model of molecular, structural, and functional adaptation to transepithelial ion transport and barrier function during embryonic development. J Biomed Biotechnol 2010: 940741, 2010.

7 Oskowitz A, Mcferrin H, Gutschow M, Carter ML, Orleans N and Orleans N: Serum-deprived human multipotent mesenchymal stromal cells (MSCs) are highly angiogenic. Stem Cell Res 6(3): 215-225, 2011.

8 Allameh A, Jazayeri M and Adelipour M: In vivo vascularization of endothelial cells derived from bone marrow mesenchymal Stem Cells in SCID Mouse Model. Cell J 18(2): 179-188, 2016.
9 Qian H, Le Blanc K and Sigvardsson M: Primary mesenchymal stem and progenitor cells from bone marrow lack expression of CD44 protein. J Biol Chem 287(31): 25795-25807, 2012.

10 Anderson P, Carrillo-Gálvez AB, García-Pérez A, Cobo M and Martín F: CD105 (Endoglin)-negative murine mesenchymal stromal cells define a new multipotent subpopulation with distinct differentiation and immunomodulatory capacities. PLoS One 8(10): 1-13, 2013.

11 Li DY, Sorensen LK, Brooke BS, Urness LD, Davis EC, Taylor DG, Boak BB and Wendel DP: Defective angiogenesis in mice lacking endoglin. Science 284(5419): 1534-1537, 1999.

12 Jin HJ, Park SK, Oh W, Yang YS, Kim SW and Choi SJ: Downregulation of $\mathrm{CD} 105$ is associated with multi-lineage differentiation in human umbilical cord blood-derived mesenchymal stem cells. Biochem Biophys Res Commun 381(4): 676-681, 2009.

13 Levi B, Wan DC, Glotzbach JP, Hyun J, Januszyk M, Montoro D, Sorkin M, James AW, Nelson ER, Li S, Quarto N, Lee M, Gurtner GC and Longaker MT: CD105 protein depletion enhances human adipose-derived stromal cell osteogenesis through reduction of transforming growth factor $\beta 1$ (TGF- $\beta 1$ ) signaling. J Biol Chem 286(45): 39497-39509, 2011.

14 Sidney LE, Branch MJ, Dunphy SE, Dua HS and Hopkinson A: Concise review: evidence for $\mathrm{CD} 34$ as a common marker for diverse progenitors. Stem Cells 32(6): 1380-1389, 2014.

15 Lin CS, Ning H, Lin G and Lue TF: Is CD34 truly a negative marker for mesenchymal stromal cells? Cytotherapy 14(10): 1159-1163, 2012.

16 Kurz H, Fehr J, Nitschke R and Burkhardt H: Pericytes in the mature chorioallantoic membrane capillary plexus contain desmin and $\alpha$-smooth muscle actin: Relevance for non-sprouting angiogenesis. Histochem Cell Biol 130: 1027-1040, 2008.

17 Hagedorn M, Balke M, Schmidt A, Bloch W, Kurz H, Javerzat $\mathrm{S}$, Rousseau B, Wilting $\mathrm{J}$ and Bikfalvi A: VEGF coordinates interaction of pericytes and endothelial cells during vasculogenesis and experimental angiogenesis. Dev Dyn 230(1): 23-33, 2004.

18 Comşa S, Ciuculescu F and Raica M: Mesenchymal stem celltumor cell cooperation in breast cancer vasculogenesis. Mol Med Rep 5(5): 1175-1180, 2012.

19 Strassburg S, Nienhueser H, Björn Stark G, Finkenzeller G and Torio-Padron N: Co-culture of adipose-derived stem cells and endothelial cells in fibrin induces angiogenesis and vasculogenesis in a chorioallantoic membrane model. J Tissue Eng Regen Med 10(6): 496-506, 2016. 